This item was submitted to Loughborough's Research Repository by the author.

Items in Figshare are protected by copyright, with all rights reserved, unless otherwise indicated.

\title{
Bloch waves of small high-Tc bipolarons
}

PLEASE CITE THE PUBLISHED VERSION

LICENCE

CC BY-NC-ND 4.0

REPOSITORY RECORD

Alexandrov, A.S.. 2019. "Bloch Waves of Small High-tc Bipolarons". figshare. https://hdl.handle.net/2134/1161. 


\title{
Bloch waves of small high-Tc bipolarons
}

\author{
A.S. Alexandrov \\ Department of Physics, Loughborough University, Loughborough LE11 \\ 3TU, United Kingdom
}

\begin{abstract}
Over the last decade several competing models of high-temperature superconductivity were proposed, most of them with short-range interactions. We review a more realistic model with strong on-site repulsive correlations, the Coulomb and strong finite-range electron-phonon interactions. Bipolarons in the model exist in the itinerant Bloch states at temperatures below about half of the characteristic phonon frequency. Depending on the ratio of the inter-site Coulomb repulsion and the polaron level shift the ground state of the model is a polaronic Fermi (or Luttinger) liquid, bipolaronic high- $T_{c}$ superconductor, or charge-segregated insulator for the strong, intermediate, and weak Coulomb repulsion, respectively. Two particular lattices are analysed in detail: a chain with the finite range electron-phonon interaction and a zig-zag ladder. Charge carriers in the ladder are superlight mobile intersite bipolarons. They propagate coherently without emission or absorption of phonons with about the same mass as single polarons. The model describes key features of the cuprates, in particular their Tc values, different isotope effects, normal state pseudogaps, and spectral functions measured in tunnelling and photoemission.
\end{abstract}

74.20.-z,74.65.+n,74.60.Mj 


\section{INTRODUCTION}

For although high-temperature superconductivity has not been yet targeted as 'the shame and despair of theoretical physics', - a label attributed to superconductivity during the first half-century after its discovery - the parlous state of current theoretical constructions has led to a current consensus that there is no consensus on the theory of high- $T_{c}$ superconductivity [1]. Our view is that the extension of the BCS theory towards the strong interaction between electrons and ion vibrations describes naturally the phenomenon, and the high temperature superconductivity exists in the crossover region of the electron-lattice interaction strength from the BCS-like to bipolaronic superconductivity [2]. Quite remarkably Bednorz and Müller noted in their original publication [3] and subsequently in their Nobel Price lecture [4], that in their ground-breaking search for high- $\mathrm{T}_{c}$ superconductivity, they were stimulated and guided by the polaron model. Their expectation [4] was that if 'an electron and a surrounding lattice distortion with a high effective mass can travel through the lattice as a whole, and a strong electron-lattice coupling exists an insulator could be turned into a high temperature superconductor'. Indeed there is now overwhelming experimental [5,6,6,7,8] and theoretical [9, 10, 11, 12, 13, 14] evidence for an exceptionally strong electron-phonon (e-ph) interaction in high temperature superconductors. Thus the theory of HTSC must include both e-ph and electron-electron Coulomb interactions as it was suggested some time ago [9]. Also one has to take into account that all oxides are highly polarizable ionic lattices. A low density of mobile carriers is unable to screen effectively the direct Coulomb electron-ion and electron-electron interactions. The layered structure of the cuprates reduces screening even further. Since the mobile carriers are confined to the copper-oxygen planes their interaction with c-axis polarized optical phonons cannot be screened, and it is particularly strong. The parameter-free estimate of the polaron binding energy, $E_{p}$ due to the long-range Fröhlich e-ph interaction puts it at about $0.5 \mathrm{eV}$ or larger in the cuprates [15].

However bipolaronic states are much heavier than band electrons since they are 'dressed' by the same lattice deformation, which bounds two polarons in a pair [9]. As a result, 
the superconducting critical temperature, $T_{c}$, being proportional to the inverse mass of a bipolaron, might be significantly reduced rather than enhanced compared with the weakcoupling BCS $T_{c}$. Because of this prejudice some objections have been raised with respect to the bipolaron theory of high-temperature superconductivity. In the present article we review a few recent models of small bipolarons with the long-range electron-phonon (e-ph) interaction capturing the essential physics of superconducting cuprates [16]. These studies prove that small bipolarons are perfectly mobile Bloch states in the cuprates which can explain their high $T_{c}$.

The electron-phonon coupling constant $\lambda$ is about the ratio of the electron-phonon interaction energy $E_{p}$ to the half bandwidth $D$ in a rigid lattice. We expect that when the coupling is strong, $\lambda>1$, all electrons in the Bloch band are "dressed" by phonons because their kinetic energy $(<D)$ is small compared with the potential energy due to a local lattice deformation caused by an electron. If phonon frequencies are very low, the local lattice deformation traps the electron. This self-trapping phenomenon was predicted by Landau [17. It has been studied in greater detail by Pekar and Fröhlich, and later on the most advanced path-integral theory of polarons was developed by Feynman and Devreese and his school in the effective mass approximation, which leads to the so-called large polaron (for more detail see Ref. [10]). The large polaron propagates through the lattice like a free electron but with the enhanced effective mass. In the strong-coupling regime, $\lambda>1$, the finite bandwidth becomes important, so that the effective mass approximation cannot be applied. The electron is called a small polaron in this regime. The self-trapping is never "complete", that is any polaron can tunnel through the lattice coherently. Only in the extreme adiabatic limit, when the phonon frequencies tend to zero, the self-trapping is complete, and the polaron motion is no longer translationally continuous. The main features of the small polaron were understood by Tjablikov [18], Yamashita and Kurosava [19], Sewell [20], Holstein and his school [21], Lang and Firsov [22], and others and described in several review papers and textbooks [23, 24, 9, 10, 25, 26, 27]. The exponential reduction of the bandwidth at large values of $\lambda$ is one of those features. The small polaron bandwidth decreases with increasing temperature 
up to a crossover region from the coherent small polaron tunneling to a thermally activated hopping. The crossover from the polaron Bloch states to the incoherent hopping takes place at temperatures $T \approx \omega_{0} / 2$ or higher, where $\omega_{0}$ is the characteristic phonon frequency. Here we show that small bipolarons are also in the itinerant Bloch states in a wide temperature region. Moreover, they propagate with about the same mass as a single polaron in particular lattices such as perovskites.

\section{SMALL POLARON BAND}

The canonical approach to a small polaron problem is based on the displacement (LangFirsov) transformation of the electron-phonon Hamiltonian [22 in the site (m) representation for electrons allowing for the summation of all diagrams including the vertex corrections,

$$
\begin{gathered}
H=\sum_{i, j} T(\mathbf{m}-\mathbf{n}) \delta_{s s^{\prime}} c_{i}^{\dagger} c_{j}+ \\
\sum_{\mathbf{q}, i} \omega_{\mathbf{q}} \hat{n}_{i}\left[u_{i}(\mathbf{q}) d_{\mathbf{q}}+H . c .\right]+\frac{1}{2} \sum_{i \neq j} V_{c}(\mathbf{m}-\mathbf{n}) \hat{n}_{i} \hat{n}_{j}+\sum_{\mathbf{q}} \omega_{\mathbf{q}}\left(d_{\mathbf{q}}^{\dagger} d_{\mathbf{q}}+1 / 2\right) .
\end{gathered}
$$

Here $T(\mathbf{m})$ is the bare hopping integral,

$$
u_{i}(\mathbf{q})=\frac{1}{\sqrt{2 N}} \gamma(\mathbf{q}) e^{i \mathbf{q} \cdot \mathbf{m}}
$$

is the matrix element of the electron-phonon interaction, $i=(\mathbf{m}, s), j=\left(\mathbf{n}, s^{\prime}\right), s=\uparrow \downarrow$, $\hat{n}_{i}=c_{i}^{\dagger} c_{i}, c_{i}, d_{\mathbf{q}}$ are the electron (hole) and phonon operators, respectively, and $N$ is the number of sites $\left(\hbar=c=k_{B}=1\right)$.

Following Lang and Firsov [22] one can apply the canonical transformation $e^{S}$ to diagonalise the Hamiltonian. The diagonalisation is exact if $T(\mathbf{m})=0($ or $\lambda=\infty)$ :

$$
\tilde{H}=e^{S} H e^{-S},
$$

where

$$
S=-\sum_{\mathbf{q}, i} \hat{n}_{i}\left[u_{i}(\mathbf{q}) d_{\mathbf{q}}-H . c .\right]
$$


The electron operator transforms as

$$
\begin{aligned}
\tilde{c}_{i} & =c_{i} \hat{X}_{i}, \\
\hat{X}_{i} & =\exp \left[\sum_{\mathbf{q}} u_{i}(\mathbf{q}) d_{\mathbf{q}}-H . c .\right],
\end{aligned}
$$

and the phonon one as

$$
\tilde{d}_{\mathbf{q}}=d_{\mathbf{q}}-\sum_{i} \hat{n}_{i} u_{i}^{*}(\mathbf{q})
$$

It follows from Eq.(5) that the Lang-Firsov canonical transformation shifts ions to new equilibrium positions. In a more general sense it changes the boson vacuum. As a result,

$$
\begin{aligned}
\tilde{H}= & \sum_{i, j} \hat{\sigma}_{i j} c_{i}^{\dagger} c_{j}-E_{p} \sum_{i} \hat{n}_{i}+ \\
& \sum_{\mathbf{q}} \omega_{\mathbf{q}}\left(d_{\mathbf{q}}^{\dagger} d_{\mathbf{q}}+1 / 2\right)+\frac{1}{2} \sum_{i \neq j} v_{i j} \hat{n}_{i} \hat{n}_{j},
\end{aligned}
$$

where

$$
\hat{\sigma}_{i j}=T(\mathbf{m}-\mathbf{n}) \delta_{s s^{\prime}} \exp \left(\sum_{\mathbf{q}}\left[u_{j}(\mathbf{q})-u_{i}(\mathbf{q})\right] d_{\mathbf{q}}-H . c .\right)
$$

is the renormalised hopping integral depending on the phonon variables, and

$$
v_{i j}=V_{c}(\mathbf{m}-\mathbf{n})-\frac{1}{N} \sum_{\mathbf{q}}|\gamma(\mathbf{q})|^{2} \omega_{\mathbf{q}} \cos [\mathbf{q} \cdot(\mathbf{m}-\mathbf{n})]
$$

is the the interaction of polarons owing to the Coulomb repulsion $V_{c}(\mathbf{m}-\mathbf{n})$ and to the local lattice deformation (the second term).

In an extreme strong coupling limit $\lambda \rightarrow \infty$ one can neglect the hopping term of the transformed Hamiltonian. The rest has analytically determined eigenstates and eigenvalues. The eigenstates $|\tilde{N}\rangle=\left|n_{i}, n_{\mathbf{q}}\right\rangle$ are classified with the polaron $n_{\mathbf{m}, s}$ and phonon $n_{\mathbf{q}}$ occupation numbers and the energy levels are

$$
E=-E_{p} \sum_{i} n_{i}+\frac{1}{2} \sum_{i \neq j} v_{i j} n_{i} n_{j}+\sum_{\mathbf{q}} \omega_{\mathbf{q}}\left(n_{\mathbf{q}}+1 / 2\right)
$$

where $n_{i}=0,1$ and $n_{\mathbf{q}}=0,1,2,3, \ldots \infty$. 
Hence, the Hamiltonian Eq.(1) in zero order with respect to the hopping describes localised polarons and independent phonons which are vibrations of ions relative to new equilibrium positions depending on the polaron occupation numbers. The phonon frequencies remain unchanged in this limit. The middle of the electronic band falls by the polaronic level shift $E_{p}$ as a result of a potential well created by the lattice deformation,

$$
E_{p}=\frac{1}{2 N} \sum_{\mathbf{q}}|\gamma(\mathbf{q})|^{2} \omega_{\mathbf{q}} .
$$

With the finite hopping term polarons tunnel in a narrow band owing to the degeneracy of the zero order Hamiltonian with respect to the site position of a single polaron in a regular lattice. To see it one can apply the perturbation theory using $1 / \lambda$ as a small parameter with

$$
\lambda \equiv \frac{E_{p}}{z T(a)},
$$

where $z$ is the coordination lattice number and $T(a)$ is the nearest-neighbour hopping integral, so that $D \approx z T(a)$. The proper (Bloch) set of $N$ degenerate zero order eigenstates of the lowest energy level $\left(-E_{p}\right)$ of the unperturbed Hamiltonian is

$$
|\mathbf{k}, 0\rangle=\frac{1}{\sqrt{N}} \sum_{\mathbf{m}} c_{\mathbf{m}}^{\dagger} \exp (i \mathbf{k} \cdot \mathbf{m})|0\rangle,
$$

$|0\rangle$ is the vacuum. By applying the textbook perturbation theory one readily calculates the lowest energy levels of the polaron in a crystal. Up to the second order in the hopping integral the result is

$$
\begin{aligned}
E(\mathbf{k}) & =-E_{p}+\epsilon_{\mathbf{k}} \\
& -\sum_{\mathbf{k}^{\prime}, n_{\mathbf{q}}} \frac{\left|\left\langle\mathbf{k}, 0\left|\sum_{i, j} \hat{\sigma}_{i j} c_{i}^{\dagger} c_{j}\right| \mathbf{k}^{\prime}, n_{\mathbf{q}}\right\rangle\right|^{2}}{\sum_{\mathbf{q}} \omega_{\mathbf{q}} n_{\mathbf{q}}}
\end{aligned}
$$

where $\left|\mathbf{k}^{\prime}, n_{\mathbf{q}}\right\rangle$ are exited states of the unperturbed Hamiltonian with one electron and at least one real phonon. The second term in Eq.(13), which is linear with respect to the bare hopping, determines the small polaron band dispersion as

$$
\epsilon_{\mathbf{k}}=\sum_{\mathbf{m}} t(\mathbf{m}) e^{-g^{2}(\mathbf{m})} \exp (-i \mathbf{k} \cdot \mathbf{m}),
$$


with the band-narrowing factor (at zero temperature)

$$
g^{2}(\mathbf{m})=\frac{1}{2 N} \sum_{\mathbf{q}}|\gamma(\mathbf{q})|^{2}[1-\cos (\mathbf{q} \cdot \mathbf{m})]
$$

The third term in Eq. (13), quadratic in $T(a)$, yields a negative k-independent correction to the polaron level shift of the order of $1 / \lambda^{2}$.

\section{SMALL BIPOLARON BAND}

The attractive energy of two small polarons, $2 D\left(\lambda-\mu_{c}\right)$ is generally larger than the polaron bandwidth in the strong-coupling regime,

$$
\lambda-\mu_{c} \gg Z^{\prime}
$$

because the bandwidth narrowing factor $Z^{\prime}$ is small

$$
Z^{\prime}=\frac{\sum_{\mathbf{m}} T(\mathbf{m}) e^{-g^{2}(\mathbf{m})} \exp (-i \mathbf{k} \cdot \mathbf{m})}{\sum_{\mathbf{m}} T(\mathbf{m}) \exp (-i \mathbf{k} \cdot \mathbf{m})} \ll 1,
$$

where $\mu_{c}$ is the Coulomb pseudopotential. When this condition is fulfilled, small bipolarons are not overlapped. Hence the polaronic Fermi liquid transforms into a Bose liquid of doublecharged carriers. Here we encounter a novel electronic state of matter, a charged Bose liquid [2.1], qualitatively different from the normal Fermi-liquid and from the BCS superfluid.

\section{A. Onsite bipolaron band}

The small parameter, $Z^{\prime} /\left(\lambda-\mu_{c}\right) \ll 1$, allows for a consistent treatment of bipolaronic systems [2,28]. Under this condition the hopping term in the transformed Hamiltonian $\tilde{H}$ is a small perturbation of the ground state of immobile bipolarons and free phonons,

$$
\tilde{H}=H_{0}+H_{\text {pert }},
$$

where

$$
H_{0}=\frac{1}{2} \sum_{i, j} v_{i j} c_{i}^{\dagger} c_{j}^{\dagger} c_{j} c_{i}+\sum_{\mathbf{q}} \omega_{\mathbf{q}}\left[d_{\mathbf{q}}^{\dagger} d_{\mathbf{q}}+1 / 2\right]
$$


and

$$
H_{\text {pert }}=\sum_{i, j} \hat{\sigma}_{i j} c_{i}^{\dagger} c_{j}
$$

Let us first discuss dynamics of onsite bipolarons, which are the ground state of the system with the Holstein-type non-dispersive e-ph interaction. The onsite bipolaron is formed if

$$
2 E_{p}>U
$$

where $U$ is the onsite Coulomb correlation energy (the so-called Hubbard $U$ ). The intersite polaron-polaron interaction Eq.(8) is purely Coulomb repulsion because the phonon mediated attraction between two polarons on different sites is zero in the Holstein model. Two or more onsite bipolarons as well as three or more polarons cannot occupy the same site because of the Pauli exclusion principle. Hence, bipolarons repel single polarons and each other. Their binding energy, $\Delta=2 E_{p}-U$, is normally larger than the polaron halfbandwidth, $\Delta \gg w=Z^{\prime} D$, so that there are no unbound polarons in the ground state. $H_{\text {pert }}$, Eq.(19), destroys bipolarons in the first order. Hence it has no diagonal matrix elements. Then the bipolaron dynamics, including superconductivity, is described by the use of a new canonical transformation $\exp \left(S_{2}\right)$ [28], which eliminates the first order of $H_{\text {pert }}$,

$$
\left(S_{2}\right)_{f p}=\sum_{i, j} \frac{\left\langle f\left|\hat{\sigma}_{i j} c_{i}^{\dagger} c_{j}\right| p\right\rangle}{E_{f}-E_{p}} .
$$

Here $E_{f, p}$ and $|f\rangle,|p\rangle$ are the energy levels and the eigenstates of $H_{0}$. Neglecting the terms of higher orders than $(w / \Delta)^{2}$ we obtain

$$
\begin{gathered}
\left(H_{b}\right)_{f f^{\prime}} \equiv\left(e^{S_{2}} \tilde{H} e^{-S_{2}}\right)_{f f^{\prime}}, \\
\left(H_{b}\right)_{f f^{\prime}} \approx\left(H_{0}\right)_{f f^{\prime}}-\frac{1}{2} \sum_{\nu} \sum_{i \neq i^{\prime}, j \neq j^{\prime}}\left\langle f\left|\hat{\sigma}_{i i^{\prime}} c_{i}^{\dagger} c_{i^{\prime}}\right| p\right\rangle\left\langle p\left|\hat{\sigma}_{j j^{\prime}} c_{j}^{\dagger} c_{j^{\prime}}\right| f^{\prime}\right\rangle \times \\
\left(\frac{1}{E_{p}-E_{f^{\prime}}}+\frac{1}{E_{p}-E_{f}}\right) .
\end{gathered}
$$

$S_{2}$ couples a localised onsite bipolaron and a state of two unbound polarons on different sites. The expression (22) determines the matrix elements of the transformed bipolaronic 
Hamiltonian $H_{b}$ in the subspace $|f\rangle,\left|f^{\prime}\right\rangle$ with no single (unbound) polarons. On the other hand the intermediate bra $\langle p|$ and ket $|p\rangle$ in Eq.(22) refer to configurations involving two unpaired polarons and any number of phonons. Hence we have

$$
E_{p}-E_{f}=\Delta+\sum_{\mathbf{q}, \nu} \omega_{\mathbf{q} \nu}\left(n_{\mathbf{q} \nu}^{p}-n_{\mathbf{q} \nu}^{f}\right)
$$

where $n_{\mathbf{q} \nu}^{f, p}$ are phonon occupation numbers $(0,1,2,3 \ldots \infty)$. This equation is an explicit definition of the bipolaron binding energy $\Delta$ which takes into account the residual intersite repulsion between bipolarons and between two unpaired polarons. The lowest eigenstates of $H_{b}$ are in the subspace, which has only doubly occupied $c_{\mathbf{m} \uparrow}^{\dagger} c_{\mathbf{m} \downarrow}^{\dagger}|0\rangle$ or empty $|0\rangle$ sites. Onsite bipolaron tunnelling is a two-step transition. It takes place via a single polaron tunneling to a neighbouring site. The subsequent tunnelling of its "partner" to the same site restores the initial energy state of the system. There are no real phonons emitted or absorbed because the bipolaron band is narrow. Hence we can average $H_{b}$ with respect to phonons. Replacing the energy denominators in the second term in Eq.(22) by the integrals with respect to time,

$$
\frac{1}{E_{p}-E_{f}}=i \int_{0}^{\infty} d t e^{i\left(E_{f}-E p+i \delta\right) t}
$$

we obtain

$$
\begin{aligned}
H_{b}= & H_{0}-i \sum_{\mathbf{m} \neq \mathbf{m}^{\prime}, s} \sum_{\mathbf{n} \neq \mathbf{n}^{\prime}, s^{\prime}} T\left(\mathbf{m}-\mathbf{m}^{\prime}\right) T\left(\mathbf{n}-\mathbf{n}^{\prime}\right) \times \\
& c_{\mathbf{m} s}^{\dagger} c_{\mathbf{m}^{\prime} s} c_{\mathbf{n} s^{\prime}}^{\dagger} c_{\mathbf{n}^{\prime} s^{\prime}} \int_{0}^{\infty} d t e^{-i \Delta t} \Phi_{\mathbf{m m}^{\prime}}^{\mathbf{n n}^{\prime}}(t),
\end{aligned}
$$

Here $\Phi_{\mathbf{m m}^{\prime}}^{\mathbf{n n}}(t)$ is a multiphonon correlator,

$$
\Phi_{\mathbf{m m}^{\prime}}^{\mathbf{n n}}(t) \equiv\left\langle\left\langle\hat{X}_{i}^{\dagger}(t) \hat{X}_{i^{\prime}}(t) \hat{X}_{j}^{\dagger} \hat{X}_{j^{\prime}}\right\rangle\right\rangle
$$

where

$$
\hat{X}_{i}(t)=\prod_{\mathbf{q}} \exp \left[u_{i}(\mathbf{q}, t) d_{\mathbf{q}}-H . c .\right]
$$

and $u_{i}(\mathbf{q}, t)=u_{i}(\mathbf{q}) e^{i \omega_{\mathbf{q}} t} . \quad \hat{X}_{i}^{\dagger}(t)$ and $\hat{X}_{i^{\prime}}(t)$ commute for any $\gamma(\mathbf{q}, \nu)=\gamma(-\mathbf{q}, \nu)$ if $\mathbf{m} \neq \mathbf{m}^{\prime}$. Also $\hat{X}_{j}^{\dagger}$ and $\hat{X}_{j^{\prime}}$ commute, if $\mathbf{n} \neq \mathbf{n}^{\prime}$, so that we can write 


$$
\begin{aligned}
\hat{X}_{i}^{\dagger}(t) \hat{X}_{i^{\prime}}(t) & =\prod_{\mathbf{q}} e^{\left.\left[u_{i}(\mathbf{q}, t)-u_{i^{\prime}}(\mathbf{q}, t)\right] d_{\mathbf{q}}-H . c .\right]}, \\
\hat{X}_{j}^{\dagger} \hat{X}_{j^{\prime}} & =\prod_{\mathbf{q}} e^{\left.\left[u_{j}(\mathbf{q})-u_{j^{\prime}}(\mathbf{q})\right] d_{\mathbf{q}}-H . c .\right]}
\end{aligned}
$$

Applying twice the identity

$$
e^{\hat{A}+\hat{B}}=e^{\hat{A}} e^{\hat{B}} e^{-[\hat{A}, \hat{B}] / 2},
$$

yields

$$
\begin{aligned}
\hat{X}_{i}^{\dagger}(t) \hat{X}_{i^{\prime}}(t) \hat{X}_{j}^{\dagger} \hat{X}_{j^{\prime}}= & \prod_{\mathbf{q}} e^{\beta^{*} d_{\mathbf{q}}^{\dagger}} e^{-\beta d_{\mathbf{q}}} e^{-|\beta|^{2} / 2} \times \\
& e^{\left[u_{i}(\mathbf{q}, t)-u_{i^{\prime}}(\mathbf{q}, t)\right]\left[u_{j}^{*}(\mathbf{q})-u_{j^{\prime}}^{*}(\mathbf{q})\right] / 2-H . c .},
\end{aligned}
$$

where

$$
\beta=u_{i^{\prime}}(\mathbf{q}, t)-u_{i}(\mathbf{q}, t)+u_{j^{\prime}}(\mathbf{q})-u_{j}(\mathbf{q}) .
$$

Finally using the average 22]

$$
\left\langle\left\langle e^{\beta^{*} d_{\mathbf{q}}^{\dagger}} e^{-\beta d_{\mathbf{q}}}\right\rangle\right\rangle=e^{-|\beta|^{2} n_{\omega}},
$$

where $n_{\omega}=\left[\exp \left(\omega_{\mathbf{q}} / T\right)-1\right]^{-1}$ is the Bose-Einstein distribution function of phonons, we find

$$
\begin{aligned}
\Phi_{\mathbf{m m}^{\prime}}^{\mathbf{n n}^{\prime}}(t)= & e^{-g^{2}\left(\mathbf{m}-\mathbf{m}^{\prime}\right)} e^{-g^{2}\left(\mathbf{n}-\mathbf{n}^{\prime}\right)} \times \\
& \exp \left\{\frac{1}{2 N} \sum_{\mathbf{q}}|\gamma(\mathbf{q})|^{2} F_{\mathbf{q}}\left(\mathbf{m}, \mathbf{m}^{\prime}, \mathbf{n}, \mathbf{n}^{\prime}\right) \frac{\cosh \left[\omega_{\mathbf{q}}\left(\frac{1}{2 T}-i t\right)\right]}{\sinh \left[\frac{\omega_{\mathbf{q}}}{2 T}\right]}\right\}
\end{aligned}
$$

where

$$
\begin{aligned}
F_{\mathbf{q}}\left(\mathbf{m}, \mathbf{m}^{\prime}, \mathbf{n}, \mathbf{n}^{\prime}\right)= & \cos \left[\mathbf{q} \cdot\left(\mathbf{n}^{\prime}-\mathbf{m}\right)\right]+\cos \left[\mathbf{q} \cdot\left(\mathbf{n}-\mathbf{m}^{\prime}\right)\right]- \\
& \cos \left[\mathbf{q} \cdot\left(\mathbf{n}^{\prime}-\mathbf{m}^{\prime}\right)\right]-\cos [\mathbf{q} \cdot(\mathbf{n}-\mathbf{m})] .
\end{aligned}
$$

Taking into account that there are only bipolarons in the subspace, where $H_{b}$ operates, we finally rewrite the Hamiltonian in terms of the creation $b_{i}^{\dagger}=c_{\mathbf{m} \uparrow}^{\dagger} c_{\mathbf{m} \downarrow}^{\dagger}$ and annihilation $b_{i}=c_{\mathbf{m} \downarrow} c_{\mathbf{m} \uparrow}$ singlet pair operators as 


$$
\begin{aligned}
H_{b}= & -\sum_{\mathbf{m}}\left[\Delta+\frac{1}{2} \sum_{\mathbf{m}^{\prime}} v^{(2)}\left(\mathbf{m}-\mathbf{m}^{\prime}\right)\right] n_{\mathbf{m}}+ \\
& \sum_{\mathbf{m} \neq \mathbf{m}^{\prime}}\left[t\left(\mathbf{m}-\mathbf{m}^{\prime}\right) b_{\mathbf{m}}^{\dagger} b_{\mathbf{m}^{\prime}}+\frac{1}{2} \bar{v}\left(\mathbf{m}-\mathbf{m}^{\prime}\right) n_{\mathbf{m}} n_{\mathbf{m}^{\prime}}\right] .
\end{aligned}
$$

There are no triplet pairs in the Holstein model, because the Pauli exclusion principle does not allow two electrons with the same spin occupy the same site. Here $n_{\mathbf{m}}=b_{\mathbf{m}}^{\dagger} b_{\mathbf{m}}$ is the bipolaron site-occupation operator,

$$
\bar{v}\left(\mathbf{m}-\mathbf{m}^{\prime}\right)=4 v\left(\mathbf{m}-\mathbf{m}^{\prime}\right)+v^{(2)}\left(\mathbf{m}-\mathbf{m}^{\prime}\right)
$$

is the bipolaron-bipolaron interaction including the direct polaron-polaron interaction $v\left(\mathbf{m}-\mathbf{m}^{\prime}\right)$ and a repulsive correction of the second order in $T(\mathbf{m})$,

$$
v^{(2)}\left(\mathbf{m}-\mathbf{m}^{\prime}\right)=2 i \int_{0}^{\infty} d t e^{-i \Delta t} \Phi_{\mathbf{m m}^{\prime}}^{\mathbf{m}^{\prime} \mathbf{m}}(t)
$$

This additional repulsion appears because a virtual hop of one of two polarons of the pair is forbidden, if the neighbouring site is occupied by another pair. The bipolaron transfer integral is of the second order in $T(\mathbf{m})$

$$
t\left(\mathbf{m}-\mathbf{m}^{\prime}\right)=-2 i T^{2}\left(\mathbf{m}-\mathbf{m}^{\prime}\right) \int_{0}^{\infty} d t e^{-i \Delta t} \Phi_{\mathbf{m m}^{\prime}}^{\mathbf{m m}^{\prime}}(t)
$$

The bipolaronic Hamiltonian, Eq.(31) describes the low-energy physics of strongly coupled electrons and phonons. We use the explicit form of the multiphonon correlator, Eq.(29), to calculate $t(\mathbf{m})$ and $v^{(2)}(\mathbf{m})$. If the phonon frequency is dispersionless, $\omega_{\mathbf{q}}=\omega_{0}$, we obtain

$$
\begin{aligned}
& \Phi_{\mathbf{m m}^{\prime}}^{\mathbf{m m}^{\prime}}(t)=e^{-2 g^{2}\left(\mathbf{m}-\mathbf{m}^{\prime}\right)} \exp \left[-2 g^{2}\left(\mathbf{m}-\mathbf{m}^{\prime}\right) e^{-i \omega_{0} t}\right] \\
& \Phi_{\mathbf{m}^{\prime} \mathbf{m}^{\prime}}^{\mathbf{m}^{\prime} \mathbf{m}}(t)=e^{-2 g^{2}\left(\mathbf{m}-\mathbf{m}^{\prime}\right)} \exp \left[2 g^{2}\left(\mathbf{m}-\mathbf{m}^{\prime}\right) e^{-i \omega_{0} t}\right]
\end{aligned}
$$

at $T \ll \omega_{0}$. Expanding the time dependent exponents in the Fourier series and calculating the integrals in Eqs.(34) and (33) yield 29]

$$
t(\mathbf{m})=-\frac{2 T^{2}(\mathbf{m})}{\Delta} e^{-2 g^{2}(\mathbf{m})} \sum_{l=0}^{\infty} \frac{\left[-2 g^{2}(\mathbf{m})\right]^{l}}{l !\left(1+l \omega_{0} / \Delta\right)}
$$

and 


$$
v^{(2)}(\mathbf{m})=\frac{2 T^{2}(\mathbf{m})}{\Delta} e^{-2 g^{2}(\mathbf{m})} \sum_{l=0}^{\infty} \frac{\left[2 g^{2}(\mathbf{m})\right]^{l}}{l !\left(1+l \omega_{0} / \Delta\right)}
$$

When $\Delta \ll \omega_{0}$, we can keep the first term only with $l=0$ in the bipolaron hopping integral, Eq.(35). In this case the bipolaron half-bandwidth $z t(\mathbf{a})$ is of the order of $2 w^{2} /(z \Delta)$. However, if the bipolaron binding energy is large, $\Delta \gg \omega_{0}$, the bipolaron bandwidth dramatically decreases proportional to $e^{-4 g^{2}} \lll 1$ in the limit $\Delta \rightarrow \infty$. However, this limit is not realistic because $\Delta=2 E_{p}-V_{c}<2 g^{2} \omega_{0}$. In a more realistic regime, $\omega_{0}<\Delta<2 g^{2} \omega_{0}$, Eq.(35) yields

$$
t(\mathbf{m}) \approx \frac{2 \sqrt{2 \pi} T^{2}(\mathbf{m})}{\sqrt{\omega_{0} \Delta}} \exp \left[-2 g^{2}-\frac{\Delta}{\omega_{0}}\left(1+\ln \frac{2 g^{2}(\mathbf{m}) \omega_{0}}{\Delta}\right)\right] .
$$

On the contrary, the bipolaron-bipolaron repulsion, Eq.(36) has no small exponent in the limit $\Delta \rightarrow \infty, v^{(2)} \propto D^{2} / \Delta$. Together with the direct Coulomb repulsion the second order $v^{(2)}$ ensures stability of the bipolaronic liquid against clustering.

\section{B. Intersite bipolaron band in a chain model}

Onsite bipolarons are very heavy for realistic values of the onsite attractive energy $2 E_{p}$ and phonon frequencies. Indeed, to bind two polarons on a single site, $2 E_{p}$ should overcome the onsite Coulomb energy, which is typically of the order of $1 \mathrm{eV}$ or higher. Optical phonon frequencies are about $0.1 \div 0.2 \mathrm{eV}$ in novel superconductors like oxides and doped fullerenes. Therefore in the framework of the Holstein model, the mass enhancement exponent of onsite bipolarons in Eq.(37), is rather large ( $\left.\gtrsim \exp \left(2 E_{p} / \omega_{0}\right)>150\right)$, so that onsite bipolarons could hardly account for high values of the superconducting critical temperature [30].

But the Holstein model is not a typical model. The Fröhlich interaction with optical phonons, which is unscreened in polaronic systems, is much stronger. This longer-range interaction leads to a lighter polaron in the strong-coupling regime. Indeed, the polaron is heavy because it has to carry the lattice deformation with it, the same deformation that forms the polaron itself. Therefore, there exists a generic relation between the polaron stabilization energy, $E_{p}$, and the renormalization of its mass, $m \propto \exp \left(\gamma E_{p} / \omega_{0}\right)$, where the numeric coefficient $\gamma$ depends on the radius of the interaction. For a short-range e-ph 
interaction, the entire lattice deformation disappears and then forms at another site, when the polaron moves between the nearest lattices sites. Therefore, $\gamma=1$ and polarons and onsite bipolarons are very heavy for the characteristic values of $E_{p}$ and $\omega_{0}$. On the contrary, in case of a long-range interaction, only a fraction of the total deformation changes every time the polaron moves and $\gamma$ could be as small as 0.25 [30]. Clearly, this results in a dramatic enlightening of the polaron since $\gamma$ enters the exponent. Thus the small polaron mass could be $\leq 10 m_{e}$ where a Holstein-like estimate would yield a huge mass $10,000 m_{e}$. The lower mass has important consequences, because lighter polarons are more likely to remain mobile and less likely to trap on impurities.

The bipolaron also becomes much lighter, if the e-ph interaction is longer-range. There are two reasons for lowering of its mass with increasing radius of the e-ph interaction. The first one is the same as in the case of a single polaron discussed above. The second reason is the possibility to form intersite bipolarons, which, in certain lattice structures, tunnell coherently already in the first-order in $T(\mathbf{m})$ [30], in contrast with onsite bipolarons, which tunnel only in the second-order, Eq.(35).

To illustrate the essential dynamic properties of bipolarons formed by the longer-range e-ph interaction let us discuss a few simplified models. Following Bonča and Trugman [32] we first consider a single bipolaron in the chain model of Ref. [31]. One can further simplify the chain model by placing ions in the interstitial sites located between Wannier orbitals of one chain, and allowing for the e-ph interaction only with the nearest neighbours of another chain, as shown in Fig.1. The Coulomb interaction is represented by the on-site Hubbard $U$ term. The model Hamiltonian is

$$
\begin{aligned}
H= & T(a) \sum_{j}\left[c_{j+1, s}^{\dagger} c_{j s}+H . c .\right]+\omega_{0} \sum_{i, j, s} g(i, j) \hat{n}_{j s}\left(d_{i}^{\dagger}+d_{i}\right)+ \\
& \omega_{0} \sum_{i}\left[d_{i}^{\dagger} d_{i}+1 / 2\right]+U \sum_{i} \hat{n}_{j \uparrow} \hat{n}_{j \downarrow}
\end{aligned}
$$

in the site representation for both electrons and phonons, where

$$
g(i, j)=g_{0}\left[\delta_{i, j}+\delta_{i, j+1}\right]
$$


and $i, j$ are integers sorting the ions and the Wannier sites, respectively. This model is referred as the extended Holstein-Hubbard model (EHHM) [32]. We can view the EHHM as the simplest model with longer range than Holstein interaction. In comparison to the Fröhlich interaction the EHHM lacks long-range tail in the e-ph interaction, but reveals the similar physical properties. In the momentum representation the model is a one-dimensional case of the generic Hamiltonian, Eq.(1), with

$$
\gamma(q)=g_{0} \sqrt{2}\left(1+e^{i q a}\right)
$$

and $\omega(q)=\omega_{0}$. Using Eqs.(10), (14) and (8) we obtain

$$
E_{p}=\frac{g_{0}^{2} \omega_{0} a}{\pi} \int_{-\pi / a}^{\pi / a} d q[1+\cos q a]=2 g_{0}^{2} \omega_{0}
$$

for the polaron level shift,

$$
g^{2}=\frac{g_{0}^{2} a}{\pi} \int_{-\pi / a}^{\pi / a} d q\left[1-\cos ^{2} q a\right]=g_{0}^{2}
$$

for the mass enhancement exponent, and

$$
\begin{aligned}
& v(0)=U-4 g_{0}^{2} \omega_{0} \\
& v(a)=-\frac{2 g_{0}^{2} \omega_{0} a}{\pi} \int_{-\pi / a}^{\pi / a} d q[1+\cos q a] \cos q a=-2 g_{0}^{2} \omega_{0}
\end{aligned}
$$

for the onsite and intersite polaron-polaron interactions, respectively. Hence the EHHM has the numerical coefficient $\gamma=1 / 2$, and the polaron mass

$$
m_{E H P}^{*} \propto \exp \left(\frac{E_{p}}{2 \omega_{0}}\right)
$$

scales as the square root of the small Holstein polaron mass, $m_{S H P}^{*} \propto \exp \left(E_{p} / \omega_{0}\right)$. In the case when $U<2 g_{0}^{2} \omega_{0}$, the onsite bipolaron has the lowest energy because $|v(0)|>|v(a)|$. In this regime the bipolaron binding energy is

$$
\Delta=4 g_{0}^{2} \omega_{0}-U
$$

Using expression (34) for the bipolaron hopping integral we obtain the bipolaron mass as 


$$
m_{E H B}^{* *} \propto \exp \left(\frac{2 E_{p}}{\omega_{0}}\right)
$$

if $\Delta \gg \omega_{0}$. It scales as $\left(m_{E H P}^{*} / m\right)^{4}$, but occurs to be much smaller than the onsite bipolaron mass in the Holstein model, $m_{S H B}^{* *} \propto \exp \left(4 E_{p} / \omega_{0}\right)$, which scales as $\left(m_{S H P}^{*} / m\right)^{4}$. In the opposite regime, when $U>2 g_{0}^{2} \omega_{0}$, the intersite bipolaron has the lowest energy. Its binding energy

$$
\Delta=2 g_{0}^{2} \omega_{0}
$$

does not depend on $U$. Different from the onsite singlet bipolaron, the intersite bipolaron has four spin states, one singlet $S=0$ and three triplet states, $S=1$, with different $z$ components of the total spin, $S_{z}=0, \pm 1$. In the chain model, Fig.1, the intersite bipolaron also tunnells only in the second order in $T(a)$, when one of the electrons within the pair hops to the left (right) and then the other follows. This tunnelling involves the multiphonon correlation function $\Phi_{j+1, j}^{j+2, j+1}$, Eq.(29),

$$
\Phi_{j+1, j}^{j+2, j+1}=e^{-2 g_{0}^{2}}
$$

Hence the intersite bipolaron mass enhancement is

$$
m_{E H B}^{* *} \propto T^{-2}(a) \exp \left(\frac{E_{p}}{\omega_{0}}\right) \propto\left(\frac{m_{E H P}^{*}}{m}\right)^{2}
$$

in the infinite Hubbard $U$ limit, $U \rightarrow \infty$. We see that the intersite bipolaron in the chain model is lighter than the onsite bipolaron, but still remains much heavier than the polaron.

\section{Superlight intersite bipolarons in a ladder}

Any realistic theory of doped ionic insulators must include both the long-range Coulomb repulsion between carriers and the strong long-range electron-phonon interaction. From theoretical standpoint, the inclusion of the long-range Coulomb repulsion is critical in ensuring that the carriers would not form clusters. Indeed, in order to form stable bipolarons, 
the el-ph interaction has to be strong enough to overcome the Coulomb repulsion at short distances. Since the el-ph interaction is long-range, there is a potential possibility for clustering. The inclusion of the Coulomb repulsion $V_{c}$ makes the clusters unstable. More precisely, there is a certain window of $V_{c} / E_{p}$ inside which the clusters are unstable but bipolarons nonetheless form. In this parameter window bipolarons repel each other and propagate in a narrow band. At a weaker Coulomb interaction the system is a charge segregated insulator, and at a stronger Coulomb repulsion the system is the Fermi liquid, or the Luttinger liquid, if it is one-dimensional.

Let us now apply a generic "Fröhlich-Coulomb" Hamiltonian, which explicitly includes the infinite-range Coulomb and electron-phonon interactions, to a particular lattice structure [33]. The implicitly present infinite Hubbard $U$ prohibits double occupancy and removes the need to distinguish the fermionic spin. Introducing spinless fermion operators $c_{\mathbf{n}}$ and phonon operators $d_{\mathbf{m} \nu}$, the Hamiltonian is written as

$$
\begin{aligned}
H= & \sum_{\mathbf{n} \neq \mathbf{n}^{\prime}} T\left(\mathbf{n}-\mathbf{n}^{\prime}\right) c_{\mathbf{n}}^{\dagger} c_{\mathbf{n}^{\prime}}+\sum_{\mathbf{n} \neq \mathbf{n}^{\prime}} V_{c}\left(\mathbf{n}-\mathbf{n}^{\prime}\right) c_{\mathbf{n}}^{\dagger} c_{\mathbf{n}} c_{\mathbf{n}^{\prime}}^{\dagger} c_{\mathbf{n}^{\prime}}+ \\
& \omega_{0} \sum_{\mathbf{n} \neq \mathbf{m}, \nu} g_{\nu}(\mathbf{m}-\mathbf{n})\left(\mathbf{e}_{\nu} \cdot \mathbf{e}_{\mathbf{m}-\mathbf{n}}\right) c_{\mathbf{n}}^{\dagger} c_{\mathbf{n}}\left(d_{\mathbf{m} \nu}^{\dagger}+d_{\mathbf{m} \nu}\right)+ \\
& \omega_{0} \sum_{\mathbf{m}, \nu}\left(d_{\mathbf{m} \nu}^{\dagger} d_{\mathbf{m} \nu}+\frac{1}{2}\right) .
\end{aligned}
$$

Here the e-ph and phonon terms are written in real space, which is more convenient in working with complex lattices, $g_{\nu}(\mathbf{m}-\mathbf{n})$ is a dimensionless force acting between the electron on site $\mathbf{n}$ and the displacement of ion $\mathbf{m}, \mathbf{e}_{\mathbf{m}-\mathbf{n}} \equiv(\mathbf{m}-\mathbf{n}) /|\mathbf{m}-\mathbf{n}|$ is the unit vector in the direction from the electron $\mathbf{m}$ to the ion $\mathbf{n}$, and $\mathbf{e}_{\nu}$ is the polarization vector of the phonon branch $\nu$. Atomic orbitals of an ion adiabatically follow its motion. Therefore the electron does not interact with the displacement of the ion, whose orbital it occupies, that is $g_{\nu}(0)=0$.

In general, the many-body model Eq.(48) is of considerable complexity. However, we are interested in the limit of the strong el-ph interaction. In this case, the kinetic energy is a perturbation and the model can be grossly simplified using the canonical transformation 
of Section 2, which has the following form in the Wannier representation for electrons and phonons,

$$
S=\sum_{\mathbf{m} \neq \mathbf{n}, \nu} g_{\nu}(\mathbf{m}-\mathbf{n})\left(\mathbf{e}_{\nu} \cdot \mathbf{e}_{\mathbf{m}-\mathbf{n}}\right) c_{\mathbf{n}}^{\dagger} c_{\mathbf{n}}\left(d_{\mathbf{m} \nu}^{\dagger}-d_{\mathbf{m} \nu}\right)
$$

The transformed Hamiltonian is

$$
\begin{aligned}
\tilde{H}= & e^{S} H e^{-S}=\sum_{\mathbf{n} \neq \mathbf{n}^{\prime}} \hat{\sigma}_{\mathbf{n} \mathbf{n}^{\prime}} c_{\mathbf{n}}^{\dagger} c_{\mathbf{n}^{\prime}}+\omega_{0} \sum_{\mathbf{m} \alpha}\left(d_{\mathbf{m} \nu}^{\dagger} d_{\mathbf{m} \nu}+\frac{1}{2}\right)+ \\
& \sum_{\mathbf{n} \neq \mathbf{n}^{\prime}} v\left(\mathbf{n}-\mathbf{n}^{\prime}\right) c_{\mathbf{n}}^{\dagger} c_{\mathbf{n}} c_{\mathbf{n}^{\prime}}^{\dagger} c_{\mathbf{n}^{\prime}}-E_{p} \sum_{\mathbf{n}} c_{\mathbf{n}}^{\dagger} c_{\mathbf{n}} .
\end{aligned}
$$

The last term describes the energy which polarons gain due to el-ph interaction. $E_{p}$ is the familiar polaron level shift

$$
E_{p}=\omega \sum_{\mathbf{m} \nu} g_{\nu}^{2}(\mathbf{m}-\mathbf{n})\left(\mathbf{e}_{\nu} \cdot \mathbf{e}_{\mathbf{m}-\mathbf{n}}\right)^{2}
$$

which is independent of $\mathbf{n}$. The third term on the right-hand side in Eq.(49) is the polaronpolaron interaction:

$$
v\left(\mathbf{n}-\mathbf{n}^{\prime}\right)=V_{c}\left(\mathbf{n}-\mathbf{n}^{\prime}\right)-V_{p h}\left(\mathbf{n}-\mathbf{n}^{\prime}\right)
$$

where

$$
\begin{aligned}
V_{p h}\left(\mathbf{n}-\mathbf{n}^{\prime}\right)= & 2 \omega_{0} \sum_{\mathbf{m}, \nu} g_{\nu}(\mathbf{m}-\mathbf{n}) g_{\nu}\left(\mathbf{m}-\mathbf{n}^{\prime}\right) \times \\
& \left(\mathbf{e}_{\nu} \cdot \mathbf{e}_{\mathbf{m}-\mathbf{n}}\right)\left(\mathbf{e}_{\nu} \cdot \mathbf{e}_{\mathbf{m}-\mathbf{n}^{\prime}}\right) .
\end{aligned}
$$

The phonon-induced interaction $V_{p h}$ is due to displacements of common ions by two electrons. Finally, the transformed hopping operator $\hat{\sigma}_{\mathbf{n n}^{\prime}}$ in the first term in Eq.(49) is given by

$$
\begin{aligned}
\hat{\sigma}_{\mathbf{n}^{\prime}}= & T\left(\mathbf{n}-\mathbf{n}^{\prime}\right) \exp \left[\sum _ { \mathbf { m } , \nu } \left[g_{\nu}(\mathbf{m}-\mathbf{n})\left(\mathbf{e}_{\nu} \cdot \mathbf{e}_{\mathbf{m}-\mathbf{n}}\right)\right.\right. \\
& \left.\left.-g_{\nu}\left(\mathbf{m}-\mathbf{n}^{\prime}\right)\left(\mathbf{e}_{\nu} \cdot \mathbf{e}_{\mathbf{m}-\mathbf{n}^{\prime}}\right)\right]\left(d_{\mathbf{m} \nu}^{\dagger}-d_{\mathbf{m} \nu}\right)\right] .
\end{aligned}
$$

This term is a perturbation at large $\lambda$. Here we consider a particular lattice structure (ladder), where bipolarons tunnell already in the first order in $T(\mathbf{n})$, so that $\hat{\sigma}_{\mathbf{n n}^{\prime}}$ can be averaged over phonons. When $T \lesssim \omega_{0}$ the result is 


$$
\begin{aligned}
t\left(\mathbf{n}-\mathbf{n}^{\prime}\right) & \equiv\left\langle\left\langle\hat{\sigma}_{\mathbf{n n}^{\prime}}\right\rangle\right\rangle_{p h}=T\left(\mathbf{n}-\mathbf{n}^{\prime}\right) \exp \left[-g^{2}\left(\mathbf{n}-\mathbf{n}^{\prime}\right)\right], \\
g^{2}\left(\mathbf{n}-\mathbf{n}^{\prime}\right)= & \sum_{\mathbf{m}, \nu} g_{\nu}(\mathbf{m}-\mathbf{n})\left(\mathbf{e}_{\nu} \cdot \mathbf{e}_{\mathbf{m}-\mathbf{n}}\right) \times \\
& {\left[g_{\nu}(\mathbf{m}-\mathbf{n})\left(\mathbf{e}_{\nu} \cdot \mathbf{e}_{\mathbf{m}-\mathbf{n}}\right)-g_{\nu}\left(\mathbf{m}-\mathbf{n}^{\prime}\right)\left(\mathbf{e}_{\nu} \cdot \mathbf{e}_{\mathbf{m}-\mathbf{n}^{\prime}}\right)\right] . }
\end{aligned}
$$

By comparing Eqs.(53) and Eq.(51), the mass renormalization exponent can be expressed via $E_{p}$ and $V_{p h}$ as follows

$$
g^{2}\left(\mathbf{n}-\mathbf{n}^{\prime}\right)=\frac{1}{\omega_{0}}\left[E_{p}-\frac{1}{2} V_{p h}\left(\mathbf{n}-\mathbf{n}^{\prime}\right)\right]
$$

Now phonons are "integrated out" and the polaronic Hamiltonian is

$$
\begin{gathered}
H_{p}=H_{0}+H_{\text {pert }}, \\
H_{0}=-E_{p} \sum_{\mathbf{n}} c_{\mathbf{n}}^{\dagger} c_{\mathbf{n}}+\sum_{\mathbf{n} \neq \mathbf{n}^{\prime}} v\left(\mathbf{n}-\mathbf{n}^{\prime}\right) c_{\mathbf{n}}^{\dagger} c_{\mathbf{n}} c_{\mathbf{n}^{\prime}}^{\dagger} c_{\mathbf{n}^{\prime}}, \\
H_{\text {pert }}=\sum_{\mathbf{n} \neq \mathbf{n}^{\prime}} t\left(\mathbf{n}-\mathbf{n}^{\prime}\right) c_{\mathbf{n}^{\dagger}}^{\dagger} c_{\mathbf{n}^{\prime}} .
\end{gathered}
$$

When $V_{p h}$ exceeds $V_{c}$ the full interaction becomes negative and polarons form pairs.

The overall sign and magnitude of the interaction is given by the lattice sum in Eq.(51) evaluation of which is elementary. Notice also, that according to Eq.(54) an attractive interaction reduces the polaron mass (and consequently bipolaron mass), while repulsive interaction enhances the mass. Thus, the long-range character of the el-ph interaction serves the double purpose. Firstly, it generates additional inter-polaron attraction. This additional attraction helps overcome the direct Coulomb repulsion between the polarons. Secondly, the Fröhlich interaction makes the bipolarons lighter.

The many-particle ground state of $H_{0}$ depends on the sign of the polaron-polaron interaction, the carrier density, and the lattice geometry. Here we consider the zig-zag ladder, Fig.2a, assuming that all sites are isotropic two-dimensional harmonic oscillators. For simplicity, we also adopt the nearest-neighbour approximation for both interactions, $g_{\nu}(\mathbf{l}) \equiv g$, 
$V_{c}(\mathbf{n}) \equiv V_{c}$, and for the hopping integrals, $T(\mathbf{m})=T_{N N}$ for $l=n=m=a$, and zero otherwise. Hereafter we set the lattice period $a=1$. There are four nearest neighbours in the ladder, $z=4$. Then the one-particle polaronic Hamiltonian takes the form

$$
\begin{aligned}
H_{p}= & -E_{p} \sum_{n}\left(c_{n}^{\dagger} c_{n}+p_{n}^{\dagger} p_{n}\right)+ \\
& \sum_{n}\left[t^{\prime}\left(c_{n+1}^{\dagger} c_{n}+p_{n+1}^{\dagger} p_{n}\right)+t\left(p_{n}^{\dagger} c_{n}+p_{n-1}^{\dagger} c_{n}\right)+H . c .\right],
\end{aligned}
$$

where $c_{n}$ and $p_{n}$ are polaron annihilation operators on the lower and upper sites of the ladder, respectively, Fig.2b. Using Eqs.(50) and (53) we find

$$
\begin{aligned}
E_{p} & =4 g^{2} \omega_{0}, \\
t^{\prime} & =T_{N N} \exp \left(-\frac{7 E_{p}}{8 \omega_{0}}\right), \\
t & =T_{N N} \exp \left(-\frac{3 E_{p}}{4 \omega_{0}}\right) .
\end{aligned}
$$

The Fourier transform of Eq.(56) yields

$$
\begin{gathered}
H_{p}=\sum_{k}\left(2 t^{\prime} \cos k-E_{p}\right)\left(c_{k}^{\dagger} c_{k}+p_{k}^{\dagger} p_{k}\right)+ \\
t \sum_{k}\left[\left(1+e^{i k}\right) p_{k}^{\dagger} c_{k}+\text { H.c. }\right] .
\end{gathered}
$$

A linear transformation of $c_{k}$ and $p_{k}$ diagonalises the Hamiltonian, so that the one-particle energy spectrum $E_{1}(k)$ is found from

$$
\operatorname{det}\left|\begin{array}{cc}
2 t^{\prime} \cos k-E_{p}-E_{1}(k) & t\left(1+e^{i k}\right) \\
t\left(1+e^{-i k}\right) & 2 t^{\prime} \cos k-E_{p}-E_{1}(k)
\end{array}\right|=0 .
$$

There are two overlapping polaronic bands,

$$
E_{1}(k)=-E_{p}+2 t^{\prime} \cos (k) \pm 2 t \cos (k / 2)
$$

with the effective mass $m^{*}=2 /\left|4 t^{\prime} \pm t\right|$ near their edges.

Let us now place two polarons on the ladder. The nearest neighbour interaction, Eq.(51) is $v=V_{c}-E_{p} / 2$, if two polarons are on the different sides of the ladder, and $v=V_{c}-$ $E_{p} / 4$, if both polarons are on the same side. The attractive interaction is provided via 
the displacement of the lattice sites, which are the common nearest neighbours to both polarons. There are two such nearest neighbours for the intersite bipolaron of the type $A$ or $B$, Fig.2c, but there is only one common nearest neighbour for the bipolaron $C$, Fig.2d. When $V_{c}>E_{p} / 2$, there are no bound states and the multi-polaron system is a one-dimensional Luttinger liquid. However, when $V_{c}<E_{p} / 2$ and consequently $v<0$, the two polarons are bound into an intersite bipolaron of the type $A$ or $B$.

It is quite remarkable that the bipolaron tunnelling in the ladder appears already in the first order with respect to a single-electron tunnelling. This case is different from both onsite bipolarons and from the intersite chain bipolarons discussed above, where the bipolaron tunnelling was of the second order in $T(a)$. Indeed, the lowest energy degenerate configurations $A$ and $B$ are degenerate. They are coupled by $H_{\text {pert }}$. Neglecting all higher-energy configurations, we can project the Hamiltonian onto the subspace containing $A, B$, and empty sites. The result of such a projection is a bipolaronic Hamiltonian

$$
H_{b}=\left(V_{c}-\frac{5}{2} E_{p}\right) \sum_{n}\left[A_{n}^{\dagger} A_{n}+B_{n}^{\dagger} B_{n}\right]-t^{\prime} \sum_{n}\left[B_{n}^{\dagger} A_{n}+B_{n-1}^{\dagger} A_{n}+\text { H.c. }\right],
$$

where $A_{n}=c_{n} p_{n}$ and $B_{n}=p_{n} c_{n+1}$ are intersite bipolaron annihilation operators, and the bipolaron-bipolaron interaction is dropped (see below). Its Fourier transform yields two bipolaron bands,

$$
E_{2}(k)=V_{c}-\frac{5}{2} E_{p} \pm 2 t^{\prime} \cos (k / 2)
$$

with a combined width $4\left|t^{\prime}\right|$. The bipolaron binding energy in zero order with respect to $t, t^{\prime}$ is

$$
\Delta \equiv 2 E_{1}(0)-E_{2}(0)=\frac{E_{p}}{2}-V_{c}
$$

The bipolaron mass near the bottom of the lowest band, $m^{* *}=2 / t^{\prime}$, is

$$
m^{* *}=4 m^{*}\left[1+0.25 \exp \left(\frac{E_{p}}{8 \omega_{0}}\right)\right] .
$$

The numerical coefficient $1 / 8$ ensures that $m^{* *}$ remains of the order of $m^{*}$ even at large $E_{p}$. This fact combines with a weaker renormalization of $m^{*}$, Eq.(57), providing a superlight bipolaron. 
In models with strong intersite attraction there is a possibility of clasterization. Similar to the two-particle case above, the lowest energy of $n$ polarons placed on the nearest neighbours of the ladder is found as

$$
E_{n}=(2 n-3) V_{c}-\frac{6 n-1}{4} E_{p}
$$

for any $n \geq 3$. There are no resonating states for $n$-polaron nearest neighbour configuration if $n \geq 3$. Therefore there is no first-order kinetic energy contribution to their energy. $E_{n}$ should be compared with the energy $E_{1}+(n-1) E_{2} / 2$ of far separated $(n-1) / 2$ bipolarons

and a single polaron for odd $n \geq 3$, or with the energy of far separated $n$ bipolarons for even $n \geq 4$. "Odd" clusters are stable if

$$
V_{c}<\frac{n}{6 n-10} E_{p}
$$

and "even" clusters are stable if

$$
V_{c}<\frac{n-1}{6 n-12} E_{p}
$$

As a result we find that bipolarons repel each other and single polarons at $V_{c}>\frac{3}{8} E_{p}$. If $V_{c}$ is less than $\frac{3}{8} E_{p}$ then immobile bound clusters of three and more polarons could form. One should notice that at distances much larger than the lattice constant the polaron-polaron interaction is always repulsive, and the formation of infinite clusters, stripes or strings is impossible [34. Combining the condition of bipolaron formation and that of the instability of larger clusters we obtain a window of parameters

$$
\frac{3}{8} E_{p}<V_{c}<\frac{1}{2} E_{p},
$$

where the ladder is a bipolaronic conductor. Outside the window the ladder is either charge segregated into finite-size clusters (small $V_{c}$ ), or it is a liquid of repulsive polarons (large $V_{c}$ ).

\section{CONCLUSIONS}

It was analytically establshed a long time ago [18,21,22] that small polarons are itinerant quasiparticles existing in the Bloch states at temperatures below the characteristic phonon 
frequency for any strength of the electron-phonon coupling. Here we have reviewed more recent studies of small bipolarons in ionic lattices which show that the long-range Fröhlich interaction leads to relatively light intersite small bipolarons with the atomic size of the wave function, large binding energy and a large size of the phonon cloud. They are Bloch waves with the effective mass, which is smaller by a few orders of magnitude than the mass of onsite bipolarons in the nondispersive Holstein model. As discussed in a few original papers, reviews and books the bipolaron theory describes $T_{c}$ of many cuprates without any fitting parameters, their non Fermi-liquid normal state and the non-BCS superconducting state, and predicts single-particle spectral properties compatible with the tunnelling and ARPES spectroscopies of the cuprates.

The author greatly appreciates stimulating discussions with A.R. Bishop, A.M. Bratkovsky, J.T. Devreese, D.M. Eagles, Yu.A. Firsov, L.P. Gor'kov, V.V. Kabanov, P.E. Kornilovitch, W.Y. Liang, K.A. Müller, and S.A. Trugman. This work has been supported by the Leverhulme Trust (grant F/00261/H). 


\section{REFERENCES}

[1] A.S. Alexandrov and P.P. Edwards, Physica C331, 97 (2000), and references therein.

[2] A.S. Alexandrov, in 'Models and Phenomenology for Conventional and High-temperature Superconductivity' (Course CXXXVI of the Intenational School of Physics 'Enrico Fermi'), eds. G. Iadonisi, J.R. Schrieffer and M.L. Chiofalo, (IOS Press, Amsterdam), p. 309 (1998).

[3] J.G. Bednorz and K.A. Müller, Z. Phys. B64, 189 (1986).

[4] J.G. Bednorz and K.A. Müller, Angew. Chem. Int. Ed. Engl. 27, 735 (1988).

[5] G. Zhao, M.B. Hunt,H. Keller, and K.A. Müller, Nature 385, 236 (1997).

[6] A. Lanzara, P.V. Bogdanov, X.J. Zhou , S.A. Kellar, D.L. Feng, E.D. Lu, T. Yoshida, H. Eisaki, A. Fujimori, K. Kishio, J.I. Shimoyama, T. Noda, S. Uchida, Z. Hussain, Z.X. Shen, Nature 412, 510 (2001).

[7] T. Egami, J. Low Temp. Phys. 105, 791(1996).

[8] D.R. Temprano, J. Mesot, S. Janssen, K. Conder, A. Furrer, H. Mutka, and K.A. Müller, Phys. Rev. Lett. 84, 1990 (2000).

[9] A.S. Alexandrov and N.F. Mott, Rep.Prog.Phys. 57, 1197 (1994).

[10] J.T.Devreese, in Encyclopedia of Applied Physics, vol. 14, p. 383 (VCH Publishers, 1996).

[11] P.B. Allen, Nature 412, 494 (2001).

[12] L.P. Gor'kov, Journal of Superconductivity 12, 9 (1999).

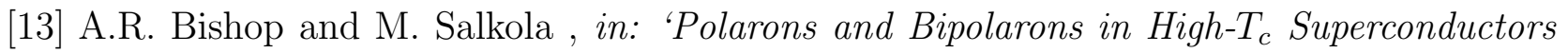
and Related Materials' eds E.K.H. Salje, A.S. Alexandrov and W.Y. Liang, Cambridge University Press, Cambridge, 353 (1995).

[14] J. Tempere, V.M. Fomin and J.T. Devreese, Solid State Commun. 101, 661 (1997).

[15] A.S. Alexandrov and A.M. Bratkovsky, Phys. Rev. Lett. 84, 2043 (2000). 
[16] for more detail see A.S. Alexandrov, 'Theory of Superconductivity: From Weak to Strong Coupling', IOP Publishers, to be published.

[17] L.D. Landau, J. Phys. (USSR) 3, 664 (1933).

[18] S.V. Tjablikov, Zh.Eksp.Teor.Fiz. 23, 381 (1952).

[19] J. Yamashita and T. Kurosawa, J.Phys.Chem.Solids 5, 34 (1958).

[20] G.L. Sewell, Phil.Mag. 3, 1361 (1958).

[21] T. Holstein, Ann.Phys. 8, 325-42; ibid p. 343 (1959).

[22] I.G. Lang and Yu.A. Firsov, Zh.Eksp.Teor.Fiz. 43, 1843 (1962) ( Sov.Phys.JETP 16, 1301 $(1963))$.

[23] J.Appel, in Solid State Physics, eds. F. Seitz, D. Turnbull and H. Ehrenreich, Academic Press 21 (1968).

[24] Yu.A. Firsov (ed), Polarons, Nauka (Moscow) (1975).

[25] H. Böttger and V.V. Bryksin, 'Hopping Conduction in Solids', Academie-Verlag Berlin (1985).

[26] G.D. Mahan, 'Many Particle Physics', Plenum Press, (1990).

[27] A.S. Alexandrov and N.F. Mott, 'Polarons and Bipolarons', World Scientific (1995); 'High Temperature Superconductors and Other Superfluids', Taylor and Francis, London (1994).

[28] A.S. Alexandrov and J. Ranninger, Phys. Rev. B 23, 1796 (1981).

[29] A.S. Alexandrov and V.V. Kabanov, Sov. Phys. Solid State, 28, 631 (1986).

[30] A.S. Alexandrov, Phys. Rev. B53, 2863 (1996).

[31] A.S. Alexandrov and P.E. Kornilovitch, Phys. Rev. Lett. 82, 807 (1999).

[32] J. Bonca and S.A. Trugman, Phys. Rev. B 64, 094507 (2001). 
[33] A.S. Alexandrov and P.E. Kornilovitch, J. Phys.: Condens. Matter 14 (2002) 5337.

[34] A.S. Alexandrov and V.V. Kabanov, JETP Lett. 72,569 (2000).

\section{Figure captions}

Fig.1. Simplified chain model with two electrons on the chain interacting with nearestneighbour ions of another chain. Second-order intersite bipolaron tunnelling is shown by arrows.

Fig.2. One-dimensional zig-zag ladder. (a) Initial ladder with the bare hopping amplitude $T(a)$. (b) Two types of polarons with their respective deformations. (c) Two degenerate bipolaron

configurations A and B. (d) A different bipolaron configuration C, which energy is higher than that of $\mathrm{A}$ and $\mathrm{B}$. 


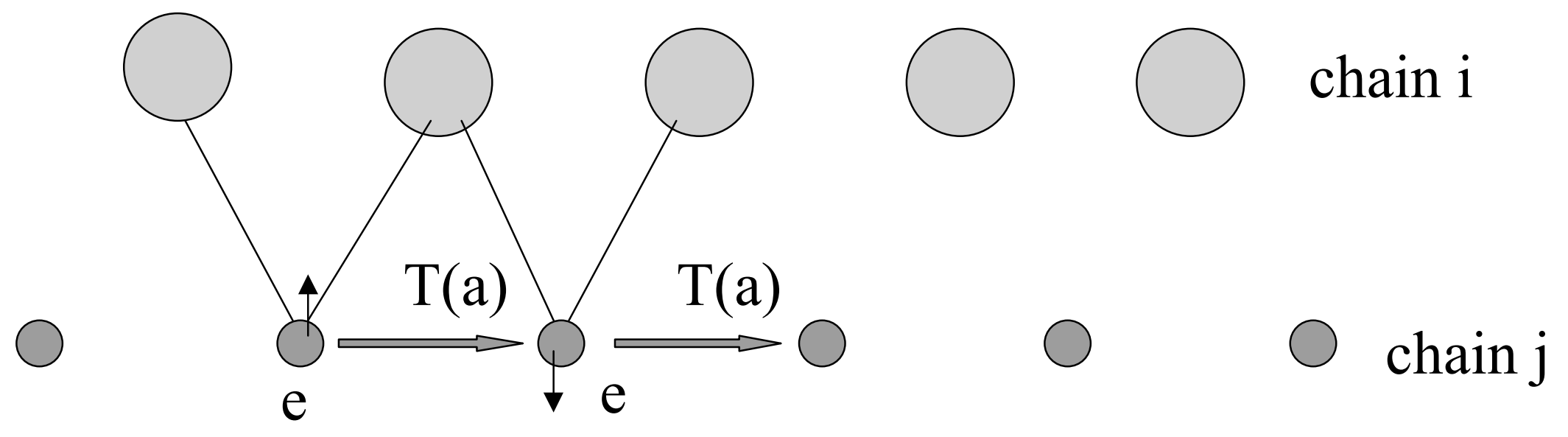




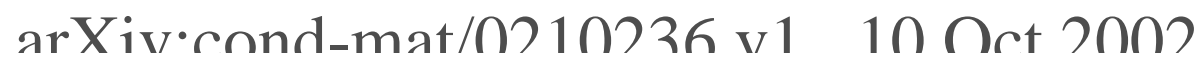
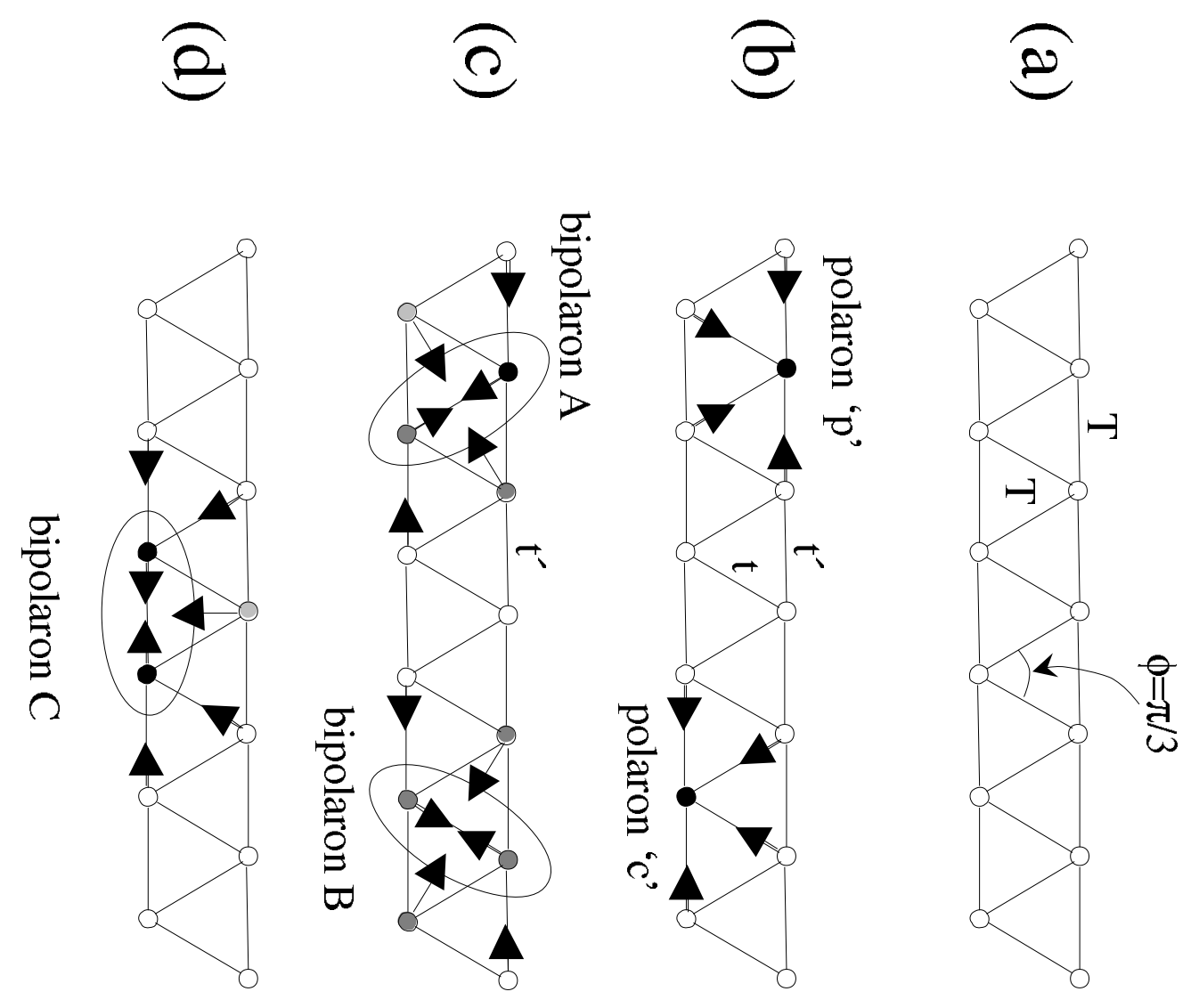